\title{
Management of Andersson Lesion in Ankylosing Spondylitis Using the Posterior-Only Approach: A Case Series of 18 Patients
}

\author{
Ismail Shaik ${ }^{1}$, Shekhar Yeshwant Bhojraj ${ }^{1}$, Gautam Prasad ${ }^{2}$, Premik Bhupendra Nagad ${ }^{1}$, \\ Priyank Mangaldas Patel ${ }^{1}$, Aaditya Dattatreya Kashikar ${ }^{1}$, Nishant Kumar ${ }^{1}$ \\ ${ }^{1}$ Department of Spine Surgery, Wockhardt Hospitals Limited, Mumbai, India \\ ${ }^{2}$ Department of Spine Surgery, Paras HMRI Hospital, Patna, India
}

Study Design: This retrospective study was conducted including 18 patients who underwent posterior-only stabilization and fusion procedure for pseudoarthrosis in the ankylosed spine from October 2007 to May 2015.

Purpose: This study aimed to describe the treatment outcomes in 18 patients with Andersson lesion (AL) who were managed using the posterior-only approach.

Literature Review: AL is an unstable, localized, vertebral, or discovertebral lesion of the spine. It is observed in patients with ankylosing spondylitis. The exact etiology of this disorder remains unclear, and the treatment guidelines are not clearly described.

Methods: We analyzed 18 patients with AL who were treated with posterior long segment spinal fusion without any anterior interbody grafting or posterior osteotomy. Pre- and postoperative radiography, computed tomography, and recent follow-up images were examined. The pre- and postoperative Visual Analog Scale score and the Oswestry Disability Index score were evaluated for all patients. Whiteclouds' outcome analysis criteria were applied at the follow-up. Moreover, at study completion, patient feedback was collected; all the patients were asked to provide their opinion regarding the surgery and were asked whether they would recommend this procedure to other patients and them self undergo the same procedure again if required.

Results: The most common site was the thoracolumbar junction. The symptom duration ranged from 1 month to 10 years preoperatively. Most patients experienced fusion by the end of 1 year, and the fusion mass could be observed as early as 4 months. Pseudoarthrosis void of up to $2.5 \mathrm{~cm}$ was noted to be healed in subsequent imaging. In addition, clinically, the patients reported good symptomatic relief. No patient required revision surgery. Whiteclouds' outcome analysis score at the latest follow-up revealed goodto-excellent outcomes in all patients.

Conclusions: ALs can be treated using the posterior-only approach with long segment fixation and posterior spinal fusion. This is a safe, simple, and quick procedure that prevents the morbidity of anterior surgery.

Keywords: Andersson lesion; Ankylosing spondylitis; Pseudoarthrosis; Spinal fractures; Posterior-only approach

Received Jan 9, 2018; Revised Mar 19, 2018; Accepted Apr 17, 2018

Corresponding author: Ismail Shaik

Department of Spine Surgery, Wockhardt Hospitals Limited, Wani House, Near Wadala Naka, Mumbai Agra Road, Nashik-422001, Maharashtra, India

Tel: +91-9049138562, E-mail: ismailwithsmile@gmail.com 


\section{Introduction}

Andersson lesion (AL) is a discovertebral lesion observed in patients with ankylosing spondylitis (AS). It was first described by Andersson [1] in 1937. Cawley et al. [2] have categorized these lesions into three main groups according to whether the involvement of the discal surface of the vertebral rim (types $\mathrm{A}$ and $\mathrm{B}$ ), the cartilaginous part of the vertebral end plate (types $\mathrm{C}$ and $\mathrm{D}$ ), or both (type E) in the lesion. The instability of such lesions progressively increases from type A to type E. These are unstable 3-column lesions resulting in pseudoarthrosis in ankylosed spine.

The prevalence rate of such lesions ranges from $1 \%$ to $28 \%[3,4]$. Contrary to the commonly experienced dull, aching, diffuse back pain in AS that aggravates with inactivity and improves with mobility, the pain of AL is sharp, localized, worsens with activity, and improves with rest. Although all the regions of the ankylosed spine are susceptible, the most commonly affected site is the thoracolumbar junction. This could be attributable to the fact that this region is a transitional zone with high stress risers.

Various theories have been proposed to explain the etiology of $\mathrm{AL}$, and the most common hypothesis involves traumatic (usually trivial) or inflammatory causes. Bron et al. [5] have attempted to summarize the etiology into the following three different groups: (1) localized lesions that always have an inflammatory origin; (2) extensive lesions without fractured posterior elements. These lesions result from a combination of inflammatory and mechanical factors (last mobile segment), are always transdiscal, and are associated with unfused facet joints; and (3) extensive lesions with fractured posterior elements resulting from mechanical factors ([stress-] fracture) with a transdiscal or transvertebral location. Lesions from groups 2 and 3 have a common final pathway and result in pseudoarthrosis with the typical appearance of the AL.

Following the study by Andersson [1], several different terms have been used to refer to these localized lesions of the spine, including AL, 'discovertebral lesion,' 'vertebral lesion,' 'destructive vertebral lesion,' 'spondylodiscitis,' 'discitis,' 'diskitis,' 'sterile diskitis,' 'pseudoarthrosis,' or '(stress-) fracture.' The use of so many different terms to describe ALs in patients with AS reflects the ongoing debate regarding the exact etiology of these spinal lesions [6].

Patients with ankylosed spine are prone to fractures due to early-onset osteoporosis. This osteoporosis is sec- ondary to autoimmune inflammation that results in low bone turnover [7]. Repeated stress and biomechanical instability makes such fractures act as unstable long-bone fractures (long lever arm) that progress to non-union, resulting in hypertrophic pseudoarthrosis, AL.

The histopathological appearance of this lesion is similar to that of chronic inflammation without any bacterial/ tubercular infection. The two adjacent ankylosed segments of the spine behave as a long bone. The histology is consistent with pseudoarthrosis with hemorrhage, fibrous tissue, small amounts of callus, and sclerosis of the adjacent vertebral bone. This microscopic picture is similar to that of non-union of the long bone, giving this lesion the name pseudoarthrosis [8].

Early ALs may often be missed on plain radiography. A high degree of suspicion is required for an early diagnosis. Computed tomography (CT) scan and magnetic resonance imaging (MRI) can aid the diagnosis. The radiological features can mimic pyogenic or tuberculous infection (especially in high endemic areas) or metastasis $[9,10]$.

Limited studies have investigated whether such lesions should be managed conservatively or be operated. Various surgical approaches and strategies, such as anterior spinal fusion, combined anterior and posterior spinal fusion, short segment posterior spinal fusion, long segment posterior spinal fusion, and circumferential fusion by all posterior technique have been described [11-18]. However, no consensus has been achieved regarding the approach or the fusion criteria, such as the levels of fusion and bone grafting.

Here, we describe the cases of 18 patients who had ALs and were treated with posterior long segment spinal fusion without anterior interbody grafting or osteotomy at the level of lesion for the correction of the kyphotic deformity.

\section{Materials and Methods}

In this case series, we included 18 patients who were operated in our unit from October 2007 to May 2015 (Table 1). The study population included one woman and 17 men, with an average age at the time of operation of 54.6 years (range, 30-79 years). Three patients were operated for neurological deficit with instability pain, while the other 15 only had progressive instability pain unresponsive to conservative treatment. The average duration of preoperative symptoms was 23 months (range, 1-120 months). 

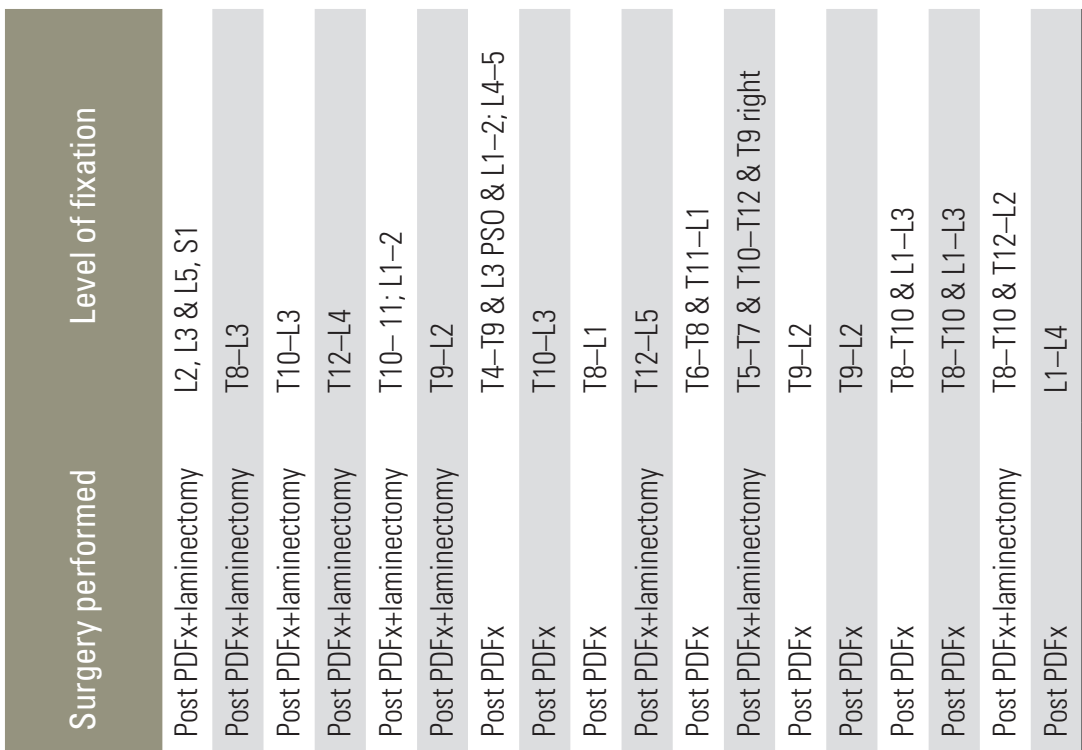

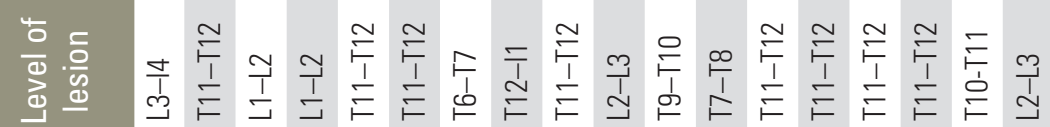
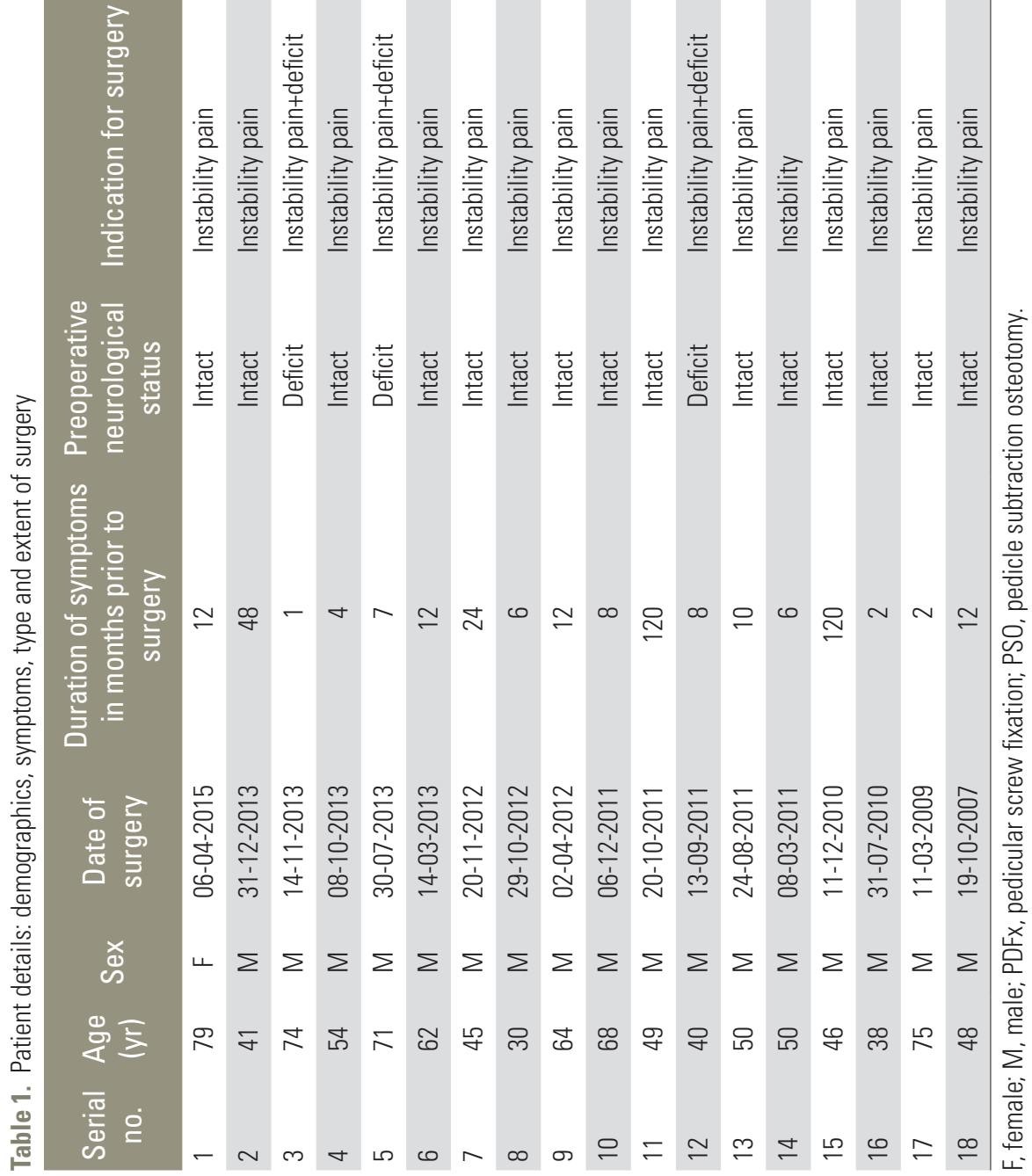


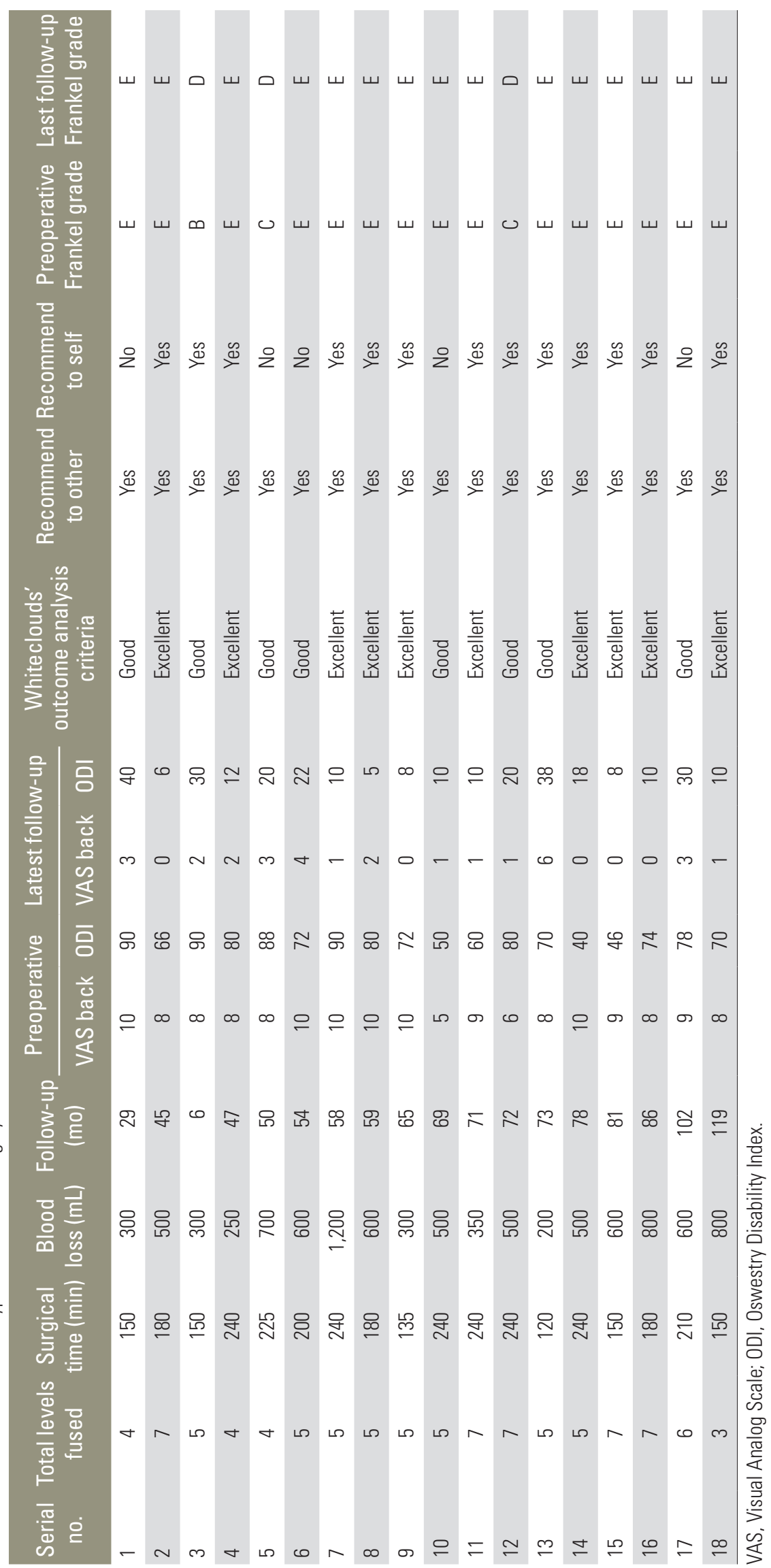


The patients were investigated using conventional radiology (radiography), CT scan, and MRI. All the patients exhibited radiological features of ankylosed 'bamboo' spine, suggestive of severe advanced AS. CT scan helped us understand the bony anatomy of the lesion, including the extent of pseudoarthrosis and deformity. The MRI scan revealed the spinal cord condition with respect to the amount of compression and damage. Thus, both CT scan and MRI scan help in surgical planning. Relevant preoperative blood investigations were performed from the anesthesia point of view.

We categorized the ALs, based on their location, as thoracic (T1-T10), thoracolumbar (T11-L2), and lumbar (L3-L5). Consequently, in this case series, we had 12 patients with ALs in the thoracolumbar region (66.67\%), more specifically at the T11-T12 level. Three patients had ALs in the thoracic region, while three had ALs in the lumbar region (16.67\% each).

We recorded the preoperative back pain levels using the Visual Analog Scale (VAS), the patient's function level using the Oswestry Disability Index (ODI), and the neurological status using Frankel grading [19].

We planned in situ posterior spinal fusion using the pedicular screw system for all patients with whatever positional correction achieved in prone positioning. The spine was exposed with a posterior midline incision; the exposure extended laterally up to the costotransverse junction in the thoracic spine and the transverse process in lumbar spine. The levels of fusion were usually 2 or 3 levels above and below the AL with an attempt to instrument the vertebra subjacent to the lesion for added stability. We did not attempt anterior stabilization with graft or cage via the anterior or posterior approach. Moreover, we did not perform osteotomy to correct the kyphosis deformity at the level of the lesion. The surgery was planned on the basis of the extent of the lesion, pseudoarthrosis, and kyphotic deformity as observed on the preoperative CT scans. Laminectomy was performed in patients with neurological deficit or those with evidence of spinal cord compression on MRI.

Empirical anti-tubercular treatment was started for $>50 \%$ of the patients in our series by their primary treating physicians. In such cases, we performed surgical biopsies of the lesion to support our diagnosis of non-infective etiology that is AS with AL.

One patient with T6-T7 AL and significant kyphosis underwent the stabilization procedure for the lesion and lumbar pedicle subtraction osteotomy (PSO) to correct the kyphosis and gain some sagittal balance in the same sitting. This patient was included in this series only for the analysis of the result at the level of the AL.

The patients were mobilized after surgery (usually by day 4) as per the pain tolerance. They were also fitted with custom-made molded braces for at least 12 weeks postoperatively. These patients were followed up at 4-month intervals with radiography and a CT scan at 1 year postoperatively to document fusion. The patients' pain score (VAS), functional level (ODI), and neurological status (Frankel grading) were recorded at each follow-up. Whiteclouds' outcome analysis criteria were applied. The patients were asked if they would recommend a similar procedure to other patients. In addition, they were asked if they would them self agree to undergo a repeat surgery in case of recurrence. Most patients were asked this feedback in person, while others were contacted telephonically.

\section{Results}

Eighteen patients underwent the procedure from October 2007 to May 2015 for AL using the above-mentioned technique in our unit. The average surgery duration was 192.77 minutes (range, 120-240 minutes) (Table 2). The average operative blood loss was $533.33 \mathrm{~mL}$ (range, 200$1,200 \mathrm{~mL})$.

The average number of spinal segments fused was 5.33 per patient (range, 3-7 segments). Eight patients underwent decompressing laminectomy with posterior spinal fusion $(44.44 \%)$, while the remaining 10 underwent only posterior spinal fusion (55.55\%).

The surgical biopsy of the suspect lesions was microbiologically sterile. Histopathology revealed chronic, nonspecific inflammation along with hypo vascular fibrous tissue consistent with the diagnosis of pseudoarthrosis (AL).

The follow-up duration was calculated from the day of surgery. The average follow-up was 64.67 months (range, 23-119 months). One patient died during follow-up, at 6 months postoperatively, due to severe respiratory tract infection.

Anterior defects as large as $2.5 \mathrm{~cm}$ were filled up as early as 8 months post stabilization and were completely fused by 2 years (Fig. 1). Most lesions showed signs of healing by 4 months postoperatively and had completely healed at 

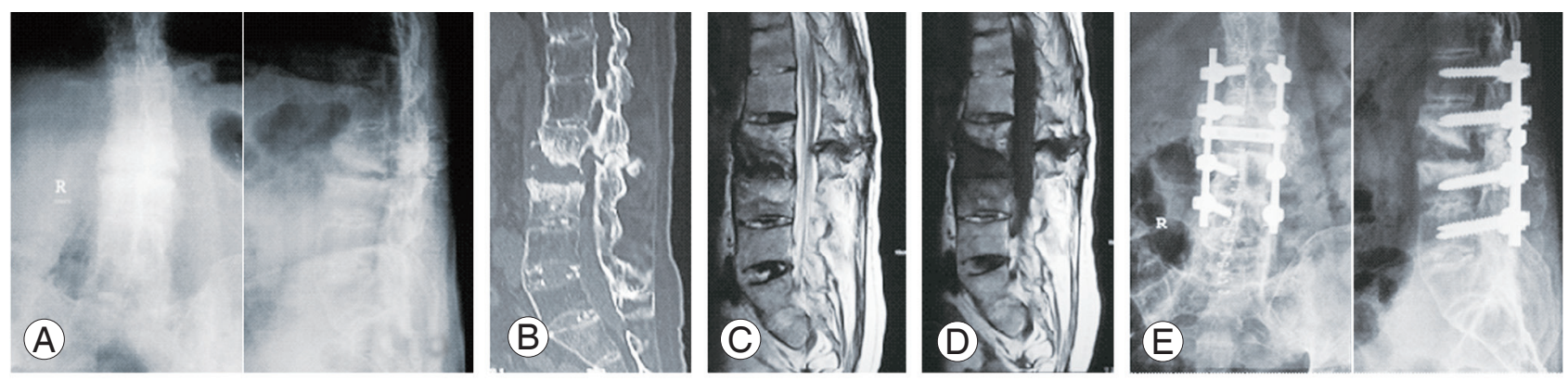

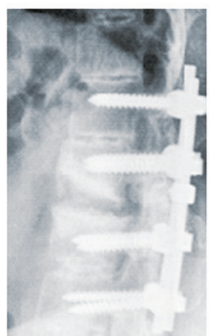

(F)
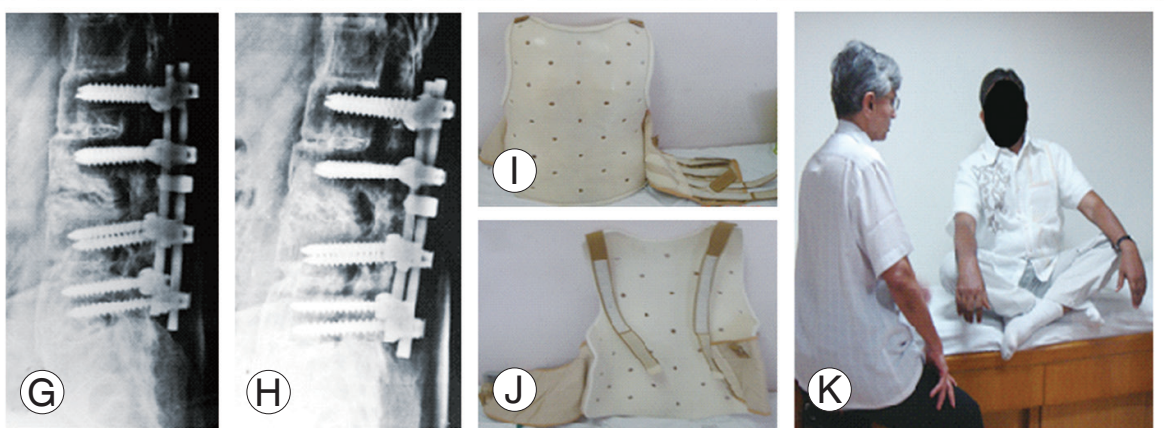

Fig. 1. L2-L3 Andersson lesion. Preoperative: radiography (A), computed tomography scan (B), MRI T2 sagittal (C), and MRI T1 sagittal (D). Postoperative: immediate after surgery 3 level posterior spinal fusion with inter transverse graft (E). Healing seen on follow-up radiography: 1 year (F), 2 years $(\mathbf{G})$, and 5 years $(\mathbf{H})$. Customized Molded Brace Jacket used as orthosis immediately after the surgery $(\mathbf{I}, \mathbf{J})$. Functional result observed at the follow-up (K). MRI, magnetic resonance imaging.
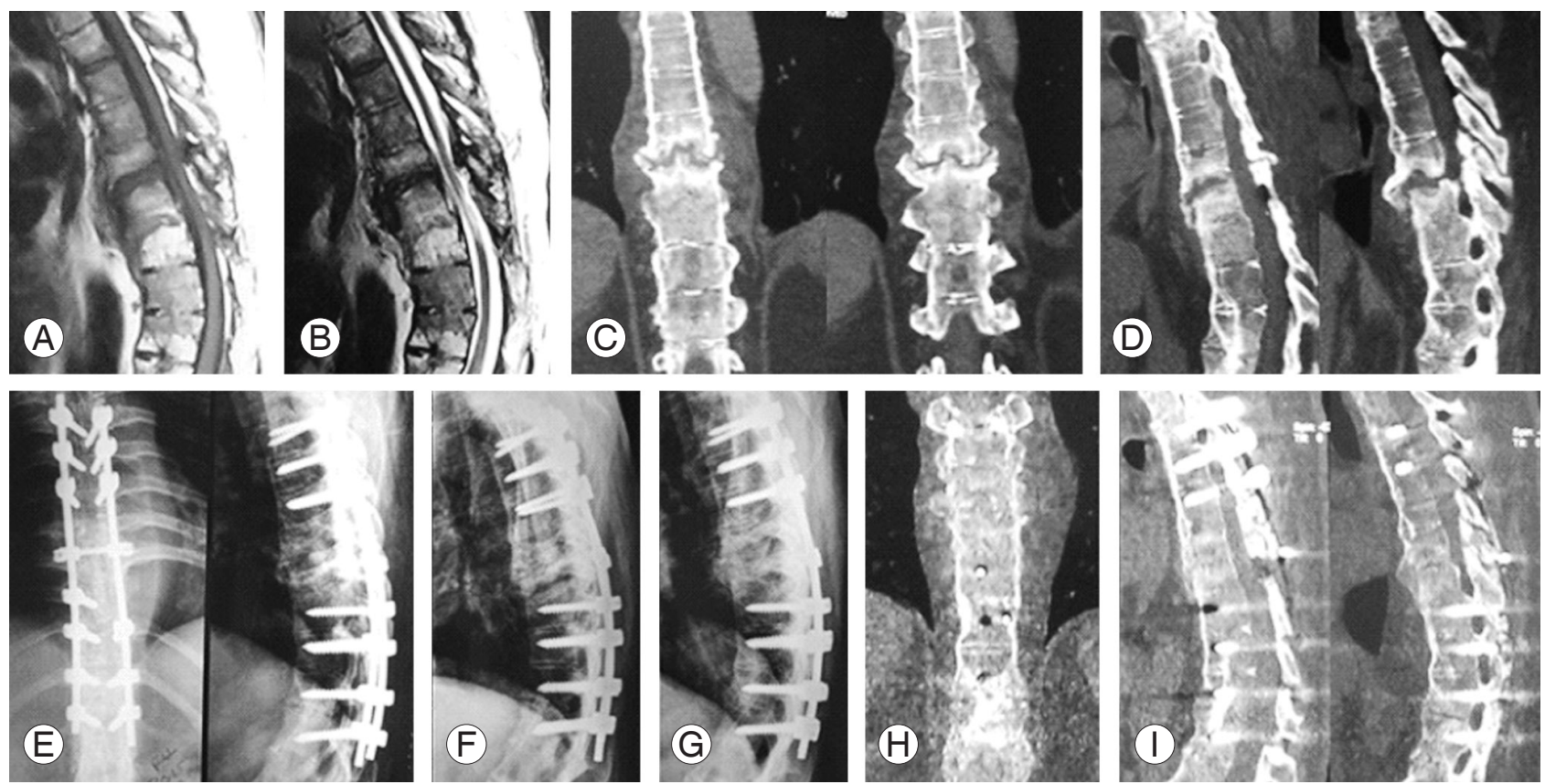

Fig. 2. T7-T8 Andersson lesion. Preoperative: MRI T1 sagittal (A), MRI T2 sagittal (B), CT scan coronal (C), and CT scan sagittal (D). Postoperative: radiography immediately after surgery (E), follow-up radiography 4 months (F), and 1 year (G); CT scan at the 1-year follow-up coronal (H) and sagittal (I), showing solid fusion. MRI, magnetic resonance imaging; CT, computed tomography.

1 year after the surgery, as observed in the CT scan (Fig. 2).

The mean VAS score decreased from a preoperative value of 8.5 (range, 5-10) to 1.6 at the final follow-up (range, $0-6$ ). The mean ODI improved from 72 (range, 40-90) to
17 (range, 5-40). Two patients with neurological deficit showed an improvement in the Frankel grade from $\mathrm{C}$ to D. One patient with a short-term neurological deficit of 2 weeks improved from Frankel grade B to grade D; howev- 
er, he succumbed to severe respiratory tract infection at 6 months postoperatively. The outcome as per Whiteclouds' outcome analysis criteria was excellent in 10 patients (55.55\%) and good in eight patients (44.44\%). All the patients said that they would recommend the procedure to other patients. Further, 13 out of 18 patients $(72.22 \%)$ were willing to undergo the procedure again if required.

Although the reduction in kyphosis deformity was measured based on clinical and radiological features, a detailed quantitative analysis of the deformity correction was not conducted in this study.

\section{Discussion}

ALs are discovertebral lesions that were first described by Andersson [1] in 1937. They were categorized into type A to type E by Cawley et al. [2] in 1972. The most severe form, type E, is unstable and involves all three columns of the spine. An AL usually occurs following trivial trauma in patients with AS and long-standing symptoms (symptoms persisting for $>10$ years). The possibility of an inflammatory etiology has also been mentioned in the literature. The incidence of such lesions varies from $1 \%$ [3] to as high as $28 \%$ [4]. Contrary to the usual dull, aching, diffuse back pain observed in AS that aggravates with inactivity and improves with mobility, the pain of an $\mathrm{AL}$ is sharp, localized, worsens with activity, and improves with rest. Although all the regions of an ankylosed spine are susceptible, the most common site is the thoracolumbar junction. This was also observed in our case series. This phenomenon could be attributable to the fact that this region is a transitional zone with high stress risers.

The histopathological picture was that of chronic inflammation without any bacterial/tubercular infection. The two adjacent ankylosed segments of the spine behaved as a long bone. The histology of type E lesions was consistent with that of pseudoarthrosis with hemorrhage, fibrous tissue, small amounts of callus, and sclerosis of the adjacent vertebral bone. There was mild inflammatory cell infiltrate that was thought to be secondary to tissue damage in the discovertebral border. Moreover, the microscopic picture was similar to that of non-union of the long bone, giving this lesion the name pseudoarthrosis [8].

Following the study by Andersson [1], several different terms have been used to refer to these localized lesions of the spine, including AL, 'discovertebral lesion,' 'vertebral lesion,' 'destructive vertebral lesion,' 'spondylodiscitis,' 'dis- citis,' 'diskitis,' 'sterile diskitis,' 'pseudarthrosis' or '(stress-) fracture.' The use of so many different terms to describe ALs in patients with AS reflects the ongoing debate regarding the exact etiology of these spinal lesions [6].

Patients with ankylosed spine are at risk of fractures due to early-onset osteoporosis. This osteoporosis is secondary to autoimmune inflammation that results in low bone turnover [7]. The fracture incidence is reportedly 5 times more than that in age-matched general population [20]. The incidence increases with age, severity of spinal ankylosis, and deformity. The ankylosis and deformity alters the biomechanics of the spine, making them vulnerable to falls and injuries. Reports also points to sex (men are at a higher risk), age, low body weight, low bone mineral density, disease duration, more extensive syndesmophyte formation, disease activity, peripheral joint involvement, spinal restriction of movement, and increased occiput to wall distance as risk factors for vertebral fractures/AL $[6,9,21,22]$. These fractures in the ankylosed spine usually involve the anterior and the posterior cortex of the vertebral body. Based on the location, they could be transdiscal or transvertebral. Such a transvertebral lesion can progress across the posterior column and lead to a classical flexion distraction traumatic injury pattern as in chance fractures [5]. Repeated stress and biomechanical instability make such fractures act as unstable long bone fractures (long lever arm) that progress to non-union that in turn give rise to hypertrophic pseudoarthrosis, AL.

Early ALs may be missed on plain radiography in most cases. Diagnosis is ensured only when there is a higher degree of suspicion. CT and MRI scan can aid the diagnosis. The radiological features can mimic pyogenic or tuberculous infection (especially in high endemic areas) or metastasis $[9,10]$. In addition, MRI is recommended for the evaluation of spinal canal encroachment and the extent of changes in the dura mater, spinal cord, nerve roots, soft tissue, and ligaments. One needs to be aware of this clinical entity, as highlighted by Dave et al. [11], where 13 out of 14 patients were diagnosed with tubercular spondylodiscitis either by their primary orthopedician or a radiologist. Even in our series, empirical anti tubercular drugs were started for more than $50 \%$ of the patients by their primary physicians.

There is no indication for biopsy in established cases of AL in AS [6]; however, we did send intra-operative tissue for histopathological and microbiological examinations in suspected cases or for those on empirical anti tubercular 
drugs. The results of these investigations were consistent with ALs with no evidence of infection in all the patients.

Healing in such lesions was described in 1985 by Marsh [12]; he described that one lesion healed with kyphosis spontaneously, while two lesions needed posterior interspinous fixation without bone grafting.

There are no clear guidelines in the literature that describe management strategies for such lesions. In our unit, for most patients, we recommend conservative management that comprises rigid immobilizing with a brace, rest, and anti-inflammatory drugs. Adjuvant therapy for AS is undertaken with the help of a rheumatologist. Although conservative treatment of such lesions has been successful, the optimal duration of therapy has not been established in previous studies [11]. We have found conservative treatment to be effective in lesions of the dorsal spine where the sternum and rib complex are intact. However, in unstable and mobile segments, such as the cervical or thoracolumbar spine, the conservative line may be ineffective [23].

Our indications for surgery were as follows: (1) neurological deficit, (2) instability threatening neurological deficit, and (3) progressively worsening pain and deformity that were similar in most studies $[11,23]$. Various surgical approaches and strategies, such as anterior spinal fusion, combined anterior and posterior spinal fusion, short segment posterior spinal fusion, long segment posterior spinal fusion, and circumferential fusion by all posterior technique have been described [11-18]. Van Royen et al. $[24,25]$ have described cases where pseudoarthrosis fixation and fusion was performed along with deformity correction at same level with success; they also described a mathematical formula for such osteotomies. Recently, a series has been published describing postoperative fracture reduction by controlled kyphosis through early mobilization following semi rigid posterior fixation with
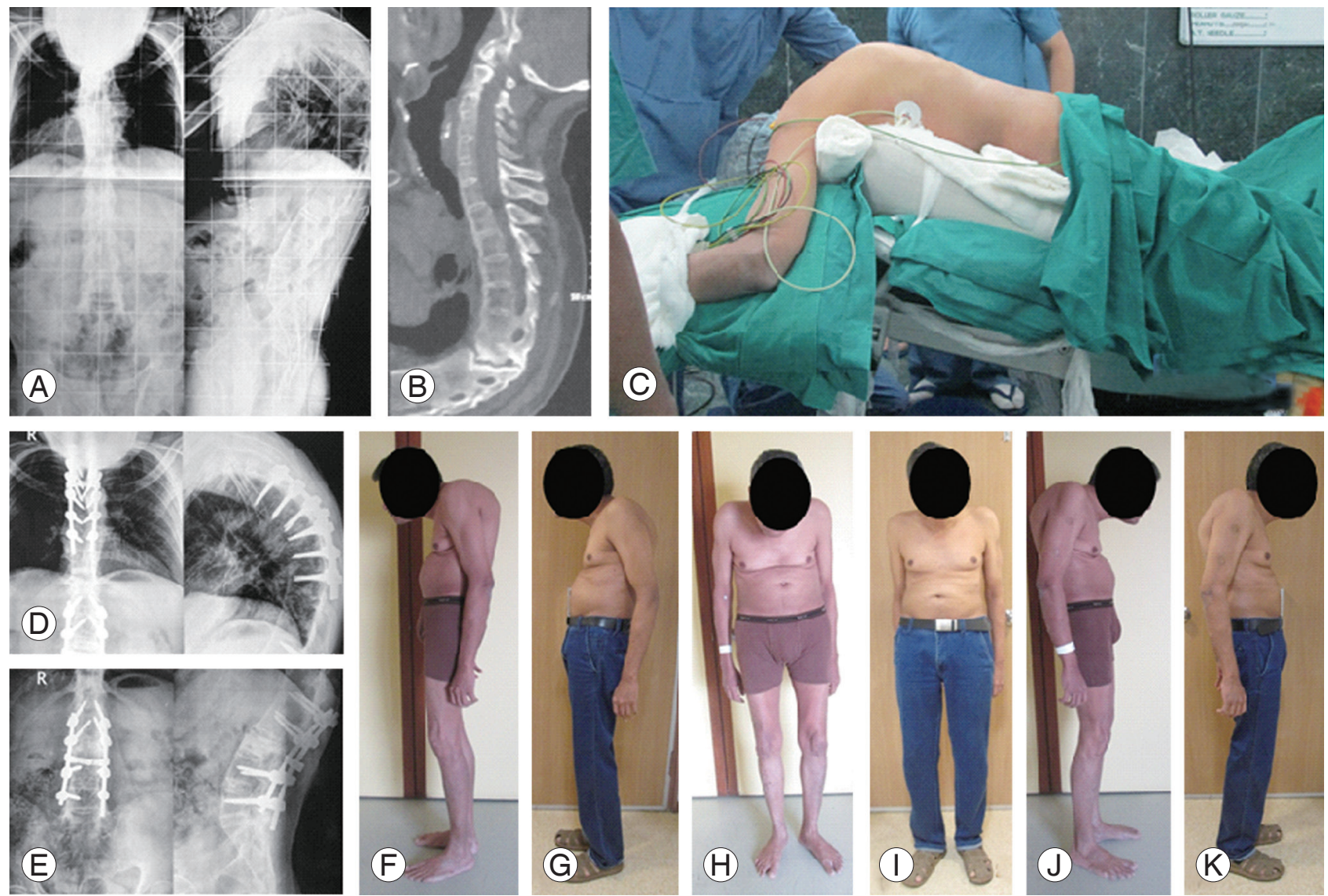

Fig. 3. T6-T7 Andersson lesion with severe kyphosis. Preoperative: radiography showing severe deformity (A); CT scan revealing the pseudoarthrosis at T6-T7 not easily visible on radiography (B). (C) Patient positioning on operation table. We did T4-T9 posterior spinal fusion for the Andersson lesion and PSO at L3 with L1L2-L4L5 pedicular screw fixation. Follow-up at 8 months: radiography fusion across Andersson lesion (D) and radiography fixation across the L3 PSO (E). Clinical outcome preoperative (F, H, J) versus follow-up at 8 months (G, I, J) showing decrease in kyphosis with improvement in the sagittal profile. CT, computed tomography; PSO, pedicle subtraction osteotomy. 
percutaneous screws [26].

Our unit previously reported a case series of 16 patients with unstable spinal lesions in AS where we used anterior only, posterior and anterior, and posterior-only approaches on case-to-case bases with good results [23]. We found that certain complications were more common in such patients. The presence of medical co-morbidities and anesthetic risks may complicate the management. Difficulties in endotracheal intubation and positioning with compromised pulmonary function add to the perioperative risks. Poor muscle mass and skin condition, osteoporotic skeleton, dural adhesions, extensive blood loss, associated neurological deficit, and problems in wound healing can complicate the intra-operative and the postoperative course. Extensive dural adhesions to the calcified ligamen- tum flavum are commonly observed; therefore, posterior exposure can result in dural tears and defects [12]. It has now been well documented that perioperative morbidity is lowest in the posterior-only approach to the spine than that in the anterior and posterior as well as the anterior only approach [27]. Pulmonary complications are common owing to restrictive lung disease that frequently occurs in long-standing AS.

Therefore, we re-considered our strategy for the management of such lesions, and this work is a presentation of the 18 cases of ALs treated using the posterior technique with long segment stabilization with no anterior debridement, bone grafting, interbody cage, or corrective posterior osteotomy [16,28]. In AS there is good bone forming and fusion tendency if stabilised adequately. We were
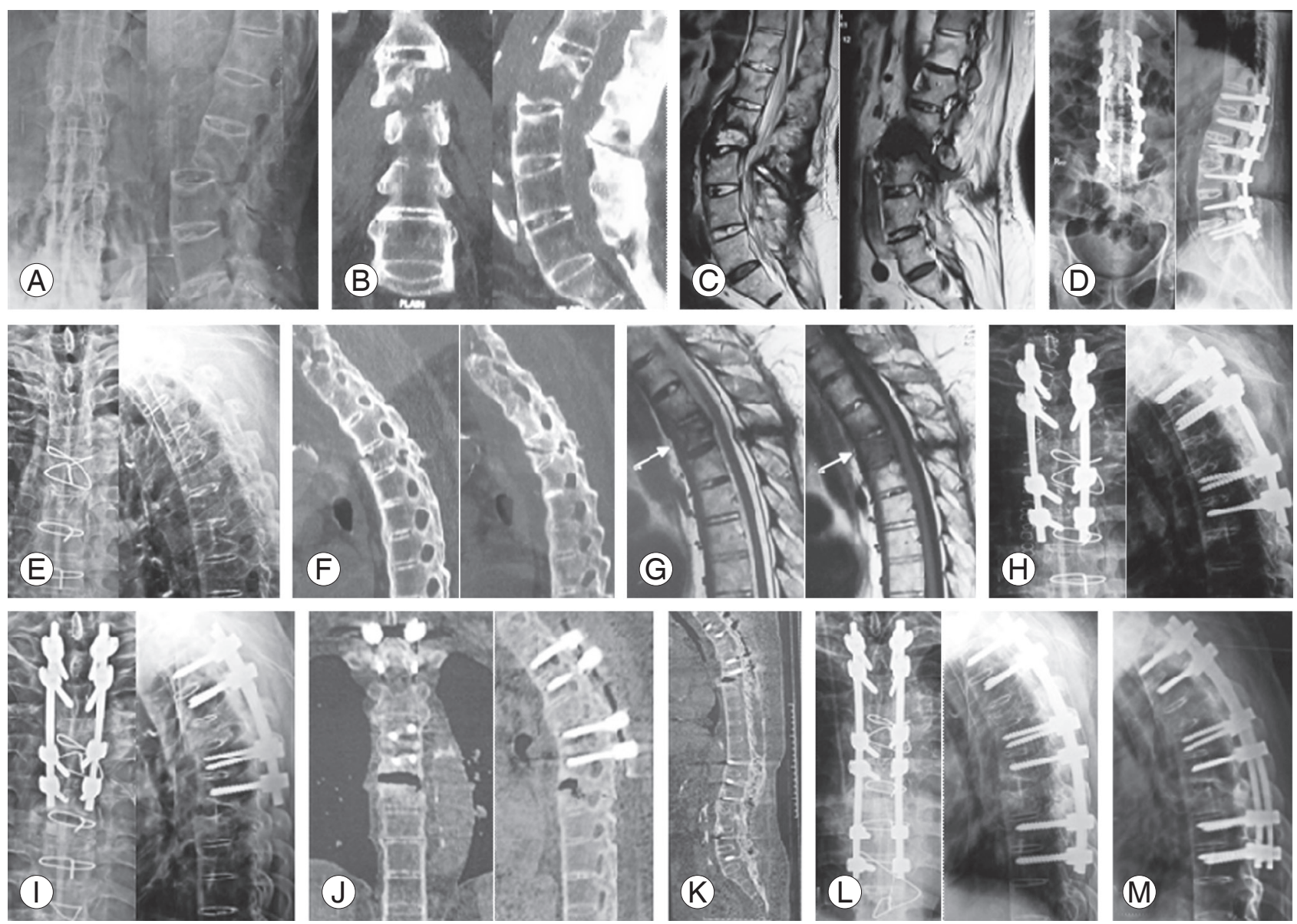

Fig. 4. L2-L3 Andersson lesion. (A) Radiography, (B) CT scan coronal \& sagittal, and (C) MRI scan T2 and T1 sagittal. (D) This patient underwent T12-L5 PSF in December 2011. He subsequently developed T4 transvertebral pseudoarthrosis: radiography (E), CT scan sagittal (F), and MRI scan T2 and T1 sagittal (G). (H) This was stabilized with T2, T3, and T5, T6 PSF in September 2015. He then developed subjacent segment T6-T7 Andersson lesion in late 2016: radiography (I) and CT scan (J) coronal and sagittal showing the fresh lesion at T6-T7. (K) Whole spine sonogram shows solid fusion at L2-L3 and T4 previous fusion levels. (L) We performed PSF till T8, T9 with rod extension in February 2017. (M) Follow-up radiography at 4 months after surgery shows evidence of callous across T6-T7. This case further proves the effectiveness in our management protocol for such Andersson lesions. CT, computed tomography; MRI, magnetic resonance imaging; PSF, posterior spinal fusion. 
encouraged by the success of an early case where an anterior void of almost $2.5 \mathrm{~cm}$ filled and fused (Fig. 1). Similar findings were reported by Chang et al. [29].

Most of our patients exhibited significant improvements in the VAS score and ODI after the surgery. One patient with T6-T7 AL and significant kyphosis underwent the stabilization procedure for the lesion and lumbar PSO to correct the kyphosis and gain some sagittal balance in the same sitting (Fig. 3). We observed good clinical and radiological outcome in this patient. This was a rare case where the AL was fixed in situ, and osteotomy for sagittal correction was performed at another level during the same sitting.

No patients required revision surgery for the same level; however, in this instance, we would like to report a participant who underwent the first surgery in December 2011 for L2-L3 ALs that was treated by our described technique of T12-L5 posterior spinal fusion. He later developed a similar lesion at $\mathrm{t} 4$ that was treated with $\mathrm{T} 2-\mathrm{T} 6$ posterior spinal fusion in September 2015. However, he then developed T6-T7 AL subjacent to previous fixation that was managed by extending the fixation to T8-T9 with rod revision in February 2017. His follow-up radiograph showed evidence of healing, and he has been clinically pain free till the time of this report (Fig. 4). All his injuries were the result of trivial trauma. This case further proves the effectiveness of our management protocol for such ALs. We believe that the inherent bone-forming tendency of AS helps in the union of AL once it has been adequately immobilized with pedicular screw fixation, as evidenced in our series. Thus, there is no concern of non-union with the use of the posterior-only approach, especially based on the case of the patient (Fig. 4) who was healed, as expected, each time. Such patients have deranged biomechanics of the spine and with added preexisting osteoporosis can be candidates for the development of adjacent segment disease owing to greater lever arm of long level fixation. Only one patient in our series developed such issues; this patient was managed as described (Fig. 4). The protocol followed by our unit while managing such adjacent segment issues involves an 'as is, when is' basis, and we do not recommend prophylactic fixation.

\section{Conclusions}

A strong clinical suspicion is required for the diagnosis of AL in AS patients. It needs to be differentiated from infective etiology in endemic areas. These lesions are unstable in nature, and their stabilization is essential for the prevention of neurological complications. We conclude that ALs can be treated using the posterior-only approach with long segment fixation and posterior spinal fusion. We present this approach as a safe, simple, and quick procedure than the more morbid anterior surgery.

We believe that a randomized controlled study would have helped clarify all the contradictory evidence; however, the low prevalence of these lesions makes it challenging to conduct a large trial. Moreover, the low prevalence is also responsible for the limited literature available on this subject; few case reports and case series have been performed. We present our findings to aid decision making in the treatment of ALs in AS.

\section{Conflict of Interest}

No potential conflict of interest relevant to this article was reported.

\section{References}

1. Andersson O. Rontgenbilden vid spondylarthritis ankylopoetica. Nord Med Tidskr 1937;14:2000-2.

2. Cawley MI, Chalmers TM, Kellgren JH, Ball J. Destructive lesions of vertebral bodies in ankylosing spondylitis. Ann Rheum Dis 1972;31:345-58.

3. Forestier J, Jacqueline F, Rotesquerol J. Ankylosing spondylarthritis with painless spinal evolution. Rev Rhum Mal Osteoartic 1951;18:486-7.

4. Jacqueline F. Disorders of bone structure and destructive lesions during ankylosing spondylarthritis. J Radiol Electrol Arch Electr Medicale 1956;37:88793.

5. Bron JL, de Vries MK, Snieders MN, van der HorstBruinsma IE, van Royen BJ. Discovertebral (Andersson) lesions of the spine in ankylosing spondylitis revisited. Clin Rheumatol 2009;28:883-92.

6. Sambrook PN, Geusens P. The epidemiology of osteoporosis and fractures in ankylosing spondylitis. Ther Adv Musculoskelet Dis 2012;4:287-92.

7. Will R, Palmer R, Bhalla AK, Ring F, Calin A. Osteoporosis in early ankylosing spondylitis: a primary pathological event? Lancet 1989;2:1483-5.

8. Calin A, Robertson D. Spondylodiscitis and pseudarthrosis in a patient with enteropathic spondyloar- 
thropathy. Ann Rheum Dis 1991;50:117-9.

9. Geusens P, Lems WF. Osteoimmunology and osteoporosis. Arthritis Res Ther 2011;13:242.

10. Dhakad U, Das SK. Andersson lesion in ankylosing spondylitis. BMJ Case Rep 2013;2013.

11. Dave BR, Ram H, Krishnan A. Andersson lesion: are we misdiagnosing it?: a retrospective study of clinicoradiological features and outcome of short segment fixation. Eur Spine J 2011;20:1503-9.

12. Marsh $\mathrm{CH}$. Internal fixation for stress fractures of the ankylosed spine. J R Soc Med 1985;78:377-9.

13. Fang D, Leong JC, Ho EK, Chan FL, Chow SP. Spinal pseudarthrosis in ankylosing spondylitis: clinicopathological correlation and the results of anterior spinal fusion. J Bone Joint Surg Br 1988;70:443-7.

14. Chen LH, Kao FC, Niu CC, Lai PL, Fu TS, Chen WJ. Surgical treatment of spinal pseudoarthrosis in ankylosing spondylitis. Chang Gung Med J 2005;28:621-8.

15. Kim KT, Lee SH, Suk KS, Lee JH, Im YJ. Spinal pseudarthrosis in advanced ankylosing spondylitis with sagittal plane deformity: clinical characteristics and outcome analysis. Spine (Phila Pa 1976) 2007;32:1641-7.

16. Wang T, Wang D, Cong Y, Yin C, Li S, Chen X. Evaluating a posterior approach for surgical treatment of thoracolumbar pseudarthrosis in ankylosing spondylitis. Clin Spine Surg 2017;30:E13-8.

17. Zhang X, Wang Y, Wu B, Hu W, Zhang Z, Wang Y. Treatment of Andersson lesion-complicating ankylosing spondylitis via transpedicular subtraction and disc resection osteotomy, a retrospective study. Eur Spine J 2016;25:2587-95.

18. Rajoli SR, Kanna RM, Aiyer SN, Shetty AP, Rajasekaran S. Circumferential fusion through allposterior approach in Andersson lesion. Asian Spine J 2017;11:444-53.

19. Frankel HL, Hancock DO, Hyslop G, et al. The value of postural reduction in the initial management of closed injuries of the spine with paraplegia and tetraplegia. I. Paraplegia 1969;7:179-92.

20. Donnelly S, Doyle DV, Denton A, Rolfe I, McCloskey EV, Spector TD. Bone mineral density and vertebral compression fracture rates in ankylosing spondylitis. Ann Rheum Dis 1994;53:117-21.

21. Vasdev V, Bhakuni D, Garg MK, Narayanan K, Jain R, Chadha D. Bone mineral density in young males with ankylosing spondylitis. Int J Rheum Dis 2011;14:6873.

22. Ghozlani I, Ghazi M, Nouijai A, et al. Prevalence and risk factors of osteoporosis and vertebral fractures in patients with ankylosing spondylitis. Bone 2009;44:772-6.

23. Badve SA, Bhojraj SY, Nene AM, et al. Spinal instability in ankylosing spondylitis. Indian J Orthop 2010;44:270-6.

24. Van Royen BJ, De Gast A, Smit TH. Deformity planning for sagittal plane corrective osteotomies of the spine in ankylosing spondylitis. Eur Spine J 2000;9:492-8.

25. Van Royen BJ, Kastelijns RC, Noske DP, Oner FC, Smit TH. Transpedicular wedge resection osteotomy for the treatment of a kyphotic Andersson lesioncomplicating ankylosing spondylitis. Eur Spine J 2006;15:246-52.

26. Lindtner RA, Kammerlander C, Goetzen M, et al. Fracture reduction by postoperative mobilisation for the treatment of hyperextension injuries of the thoracolumbar spine in patients with ankylosing spinal disorders. Arch Orthop Trauma Surg 2017;137:53141.

27. Memtsoudis SG, Vougioukas VI, Ma Y, Gaber-Baylis LK, Girardi FP. Perioperative morbidity and mortality after anterior, posterior, and anterior/posterior spine fusion surgery. Spine (Phila Pa 1976) 2011;36:186777.

28. Wang G, Sun J, Jiang Z, Cui X. The surgical treatment of Andersson lesions associated with ankylosing spondylitis. Orthopedics 2011;34:e302-6.

29. Chang KW, Tu MY, Huang HH, Chen HC, Chen YY, Lin CC. Posterior correction and fixation without anterior fusion for pseudoarthrosis with kyphotic deformity in ankylosing spondylitis. Spine (Phila $\mathrm{Pa}$ 1976) 2006;31:E408-13. 